\title{
The present situation analysis and countermeasure research of Chinese martial arts teaching in University
}

\author{
Fuchao $\mathrm{wu}^{1}$ \\ ${ }^{1}$ Taishan Medical University, China
}

Keywords: Colleges and universities, Chinese martial arts teaching, The status quo analysis, Countermeasure research.

\begin{abstract}
On Chinese martial arts teaching present situation and development trend of physical education in common colleges and universities are studied, analyzed the present situation of Chinese martial arts teaching in colleges problems, discusses the feasibility and significance of university teaching Chinese martial arts, the results show that analysis and investigations were performed and sport teachers and students in sport's teaching. It finds out that teachers' enthusiasm for teaching and students' recognition to teaching contents and values are teaching methods lack for innovation.
\end{abstract}

\section{Introduction}

Chinese martial arts is the Chinese nation in the long-term production and life practice gradually accumulated and developed a precious cultural heritage. It not only has a long history, and has a broad mass base. Chinese martial arts rich contents, various forms, unique style, are a fitness value and national traditional sports culture characteristics. Therefore, the sport has been one of the main content of physical education in schools in China.

With the constant improvement of physical education reform, with a set of physical fitness, self-defense, edify sentiment, exercise will, enhance friendship, value and function in the integral whole of Chinese martial arts sports, in physical education teaching in colleges and universities should be got further development [1]. However, we found that most of Chinese martial arts teaching in colleges and universities did not achieve the result of what we expected. Its reason mainly comes from three aspects, the first Chinese martial arts class no ball games, track and field sports courses such as well; Second difficulty Chinese martial arts teaching college students is not easy to master, and offensive and defensive class art of technology in the teaching accidents which were liable to occur, the student safety not guaranteed; The third requires a larger number of Chinese martial arts teaching equipment, various universities in the special Chinese martial arts teaching equipment are lack of corresponding number configuration. Thus causes the physical education teachers of Chinese martial arts teaching enthusiasm is not high [1]. According to this paper, the main problems of Chinese martial arts teaching in colleges, on the basis of comprehensive analysis put forward the corresponding development countermeasure, aims at colleges and universities to better carry out Chinese martial arts teaching activities, which provide reference to carry forward the national traditional sports culture.

\section{Current situation of the development of Chinese martial arts colleges and universities}

Chinese martial arts is an important part of the cultural heritage of the Chinese nation, has extensive adaptability and unique national style characteristics, combined with the characteristics of Chinese martial arts itself is action, Chinese martial arts teaching in schools has a unique meaning, it not only exercise students, character, cultivate their moral sentiment, also enrich the campus life. However, along with the rapid development of modern society and the change of social demand, Chinese martial arts teaching in colleges of the following conditions students lack of understanding of Chinese martial arts and the decrease of the recognition, lack of Chinese martial arts teachers, Chinese martial arts teaching methods obsolete backwardness, Chinese martial arts in the development of middle school is faced with serious challenges and difficulties [1], shown as Fig. 1. 


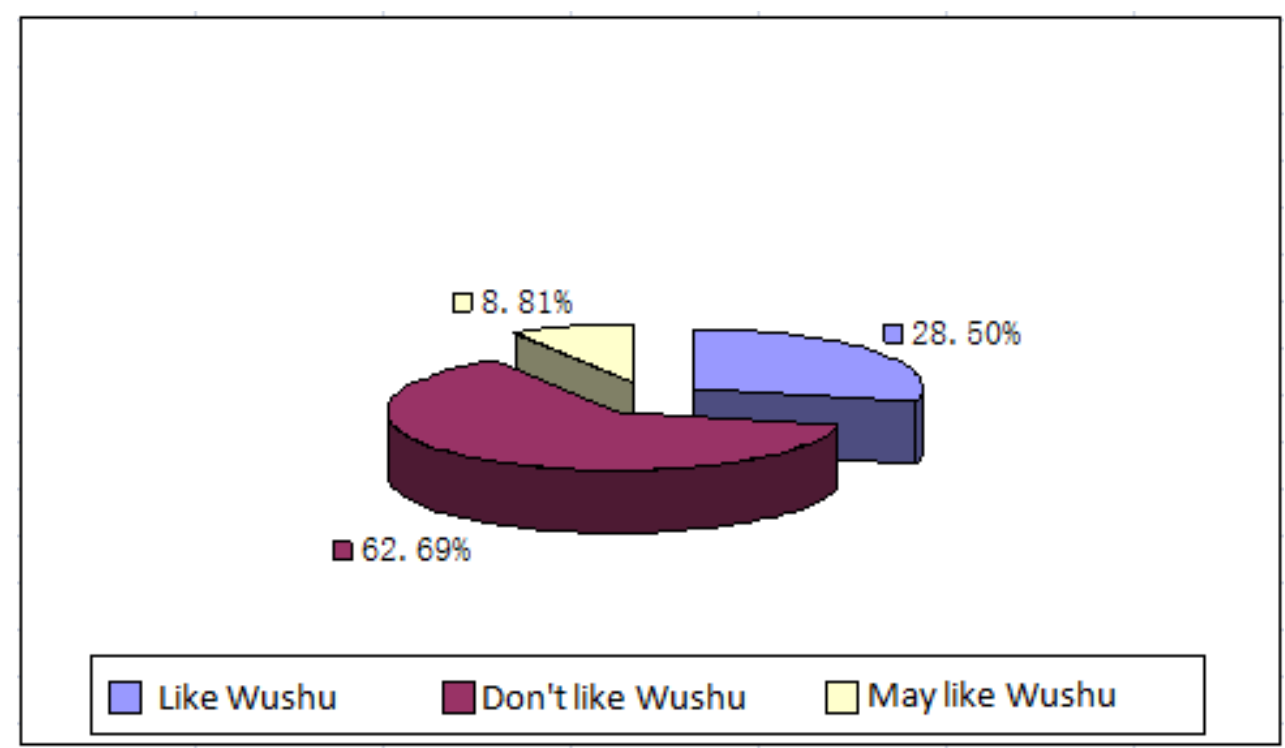

Fig. The present situation investigation of college students like Chinese martial arts Specific can induce Chinese martial arts teaching in colleges of the following conditions:

The fight club project impact of Chinese martial arts on outside. With the development of economy and culture, national culture is formed between a blend. The Chinese is the foreign countries to develop, at the same time some of the items that are foreign to enter China. The tae kwon do better development in China, its development of Chinese martial arts in the domestic launched a strong challenge.

Students' interest in Chinese martial arts. With the development of China's economy, living conditions gradually improve, for entertainment and watching sports has said, a lot of fashion project is accepted by the general college students. Relatively speaking, Chinese martial arts have some can't keep up with fashion, not interest teenagers [1]. Atmosphere, and some schools have no practicing Chinese martial arts to the promotion of the society also lack this aspect, caused some students like Chinese martial arts but nobody practice of the status quo.

An adverse effect on entrance pressure is Chinese martial arts teaching. Want to solve the problem of college Chinese martial arts development also start from the root, let the children accept Chinese martial arts since childhood, narrative, form the exercise habit. In reality, however, primary and middle school students have no time to kung fu. A no known to Chinese martial arts students enter colleges and universities, their interest can't expect him to Chinese martial arts.

Emphasis of the university and students themselves for the Chinese martial arts is not enough. From the current situation of college sports curriculum, Chinese martial arts class in universities class hour is limited in the whole training program [2]. Cause the situation today, universities and students themselves for Chinese martial arts not enough emphasis is one of the main factors.

Chinese martial arts project characteristics and evaluation system. Chinese martial arts is a technology as the main content, routines and combat as the main movement form, and pay attention to the traditional sports of internal and external and repairing [2]. The content of the Chinese martial arts are very abundant, but more complex technology, direction change. Chinese martial arts class judge how well students score on a test to give priority to the "technical" exam at the end of the semester.

\section{Chinese martial arts teaching and research universities}

Strengthen the practical application of Chinese martial arts. The present situation of Chinese martial arts teaching, strengthen the practical application of Chinese martial arts is a necessary means for the development of Chinese martial arts teaching. therefore, from the place of students interested in, in the Chinese martial arts class teaching more practical skills, fully arouse the enthusiasm of the students say, again in the process of practical teaching in Chinese martial arts education, cultivate students advocate quality of Chinese martial arts, develop a new generation of civil-military 
all-around students, with a strong will, let them to better fit into society. Let the students can not only learn skills but also learn the spirit of the Chinese martial arts culture [2]. They work in the future, inheriting the traditional culture and the spirit of the Chinese nation.

Improving Chinese martial arts teaching material. The content of the Chinese martial arts teaching should adapt to the development of society and the development of the student body and mind, optimization and improvement of Chinese martial arts teaching materials and teaching contents, changing course content "difficult, complex, partial, old" and obsessed with the present situation of book, is to make the students' interest in the new set of kung [3]. Optimization method is optimal set of Chinese martial arts skills combined with traditional culture method, the optimal set of methods of confrontational sports, reduced routine method of action, increase equipment routine with applicability and performing Chinese martial arts. Change the original boring, form a single teaching content, to ensure that the Chinese martial arts teaching content optimization on the implementation of scientific, guarantee the inheritance of Chinese traditional culture.

Increased investment in space equipment and construction. Chinese martial arts teaching can be carried out in the school, to solve the problem of space equipment is the necessary way [3]. Chinese martial arts Chinese martial arts venues and equipment are in class, the basis of the investment location equipment, Chinese martial arts teaching task is to complete the necessary condition of a good training and complete Chinese martial arts equipment can better stimulate students' interest in Chinese martial arts, to improve students' learning initiative.

\section{College Chinese martial arts teaching Suggestions}

Take the student as this, effective incentives for classroom teaching. Effective classroom incentive is from the internal factors analysis of individual students. But you can't simply use a certain internal factors to stimulate the individual [3]. Student individual specific learning environment, the size of the learning environment and atmosphere, learning, teaching requirement and the learner's behavior, and together they constitute a class of collective is incentive effect factors in the process. Teaching people to cope with students' classroom motivation play a major role.

To mobilize students' subjective initiative. Teaching to the student study independently and to set aside time to think, arouses student's enthusiasm, attract the attention of students, enhance the students' interest in learning [4]. Such as: learning the "leg" and "strike (" approach, in practice the activity for students to" play leg strike (" make action yourself, and the action method, summarize the main point, and then the teacher to explain, demonstration, evaluation, let the student control, comparison. This are deepened students understanding of the action and improve the students' ability of self-study, form the good habit of brain, more can improve the students' interest in learning, has obtained the good teaching effect.

Chinese martial arts class teaching content. Under the limited teaching resources integration and rich Chinese martial arts class teaching content, improving teaching methods, using a variety of teaching methods, for the students as much as possible the opportunity to contact and learning Chinese martial arts, in order to meet the needs of the student individuality, in learning Chinese martial arts can be carried out when a single technology, multimedia teaching the learning content [4].

So to strengthen the training of Chinese martial arts professional teachers' quality and level. In cultivating and raising the level of the Chinese martial arts professional teachers' quality and business efforts, continue to improve college Chinese martial arts teaching staff structure [3]. Because Chinese martial arts teachers' quality and professional technology level will directly affect the quality and effect of Chinese martial arts teaching, a responsible teacher can take the initiative to find the problems of Chinese martial arts teaching, and can play to subjective initiative to solve problems.

Optimize the mode of Chinese martial arts courses. Colleges and universities through reasonable improvement is Chinese martial arts curriculum (such as, special courses and elective courses) in the common course for the matching of teaching mode, to provide long-term and regular learning opportunities for students [5]. This curriculum mode can make the introversion outside class is reasonable and effective extension, to form a organic combination, such as club system, 
after-school tutoring system, etc. Build the curriculum model can not only make students outside the classroom to classroom learning Chinese martial arts techniques to master and digestion, and can be converted into the student self exercise means.

Heuristic teaching mode. It is not unilaterally by the teachers teach students the knowledge and skills, but by the students through the steps to self-discovery. Combined with guidance, pay attention to self-study alternating attaches great importance to the mental training and actual practice, strengthen the awareness of knowledge to guide students to establish roles and the development of creative thinking ability, students according to the action of learning technology the plate one to two sets of Chinese martial arts and as exam content, thus improve their ability of creative thinking [5]. The model correctly reflects the relationship between the dialectical unity of teaching and learning, emphasizes the teachers' role.

Chinese martial arts teaching with multimedia technology. With the development of modern science and technology, more and more teachers in the teaching method using high-tech and multimedia teaching are a typical representative. But in the Chinese martial arts teaching, many teachers still use the traditional demonstration, explanation, students imitate and practice and the correct teaching methods. In the simple act of teaching, teachers can adopt the method of demonstration to explain more easily finish the teaching task [6]. And in the more complex the teaching of Chinese martial arts action is very stupid teachers should not only detailed action, but also repeated front and the back side of each Angle demonstrations, to demonstrate strength is very big, to increase the teaching task and difficulty, may also make students were killed and retreat, greatly reduced the enthusiasm of students learning. Multimedia technologies into the teaching of Chinese martial arts, not only can deepen students' understanding of Chinese martial arts movements, through the video data and image data into Chinese martial arts multi-media teaching courseware in accurate action demonstration, with music, text and voice, can also realize the bilateral teaching, reduce the teacher repeated demonstration task, improve the students' learning interest and improve teaching quality [6]. Modern technology and the application of multimedia technology in the Chinese martial arts of Chinese martial arts teaching has played a good role, make up for the deficiency of the traditional Chinese martial arts teaching, improve the quality of teaching and learning effect. Multimedia teaching technology is an advanced auxiliary teaching means; we should be flexible to use multimedia, trying to explore the best combination of multimedia teaching and traditional teaching methods.

Strengthen Chinese martial arts teachers teaching quality. With the continuous development of Chinese martial arts teaching, the teaching mode and teaching systems are gradually changed, and therefore to be adapted to the development of Chinese martial arts teaching in colleges and universities in the future, in such aspects as teaching methods must be implemented to further innovation and breakthrough [7]. Teachers should change the traditional teachers teach, students learn "infusion - accept" type of single teaching mode, pay attention to the students as the main body, teacher as the leading factor of the teaching mode, to develop students' creativity; Teachers' teaching attitude, always lead by example, set a high spirit, has the vigor, serious teaching attitude; Teachers should take various methods of combining the teaching method, promote students autonomous learning, cooperative learning and inquiry learning and Chinese martial arts education carried out in the Chinese martial arts teaching, not only to practice Chinese martial arts class, should also increase Chinese martial arts theory teaching hours, make the students understand the ancient Chinese culture special connotation of Chinese martial arts, cultivate correct outlook on life, values, moral values, so that the students can use what you have learned law's' to guide their words and deeds in actual life.

Chinese martial arts teaching content reform, improve the students' learning interest. Chinese martial arts teaching lack of new theories, new methods, and some teaching materials have been out of date, and the pertinence is not strong. So the reform of Chinese martial arts teaching content, practical and high quality of Chinese martial arts teaching materials published is imperative. In view of the public physical education students professional sports is not strong, the first thing to like Chinese martial arts activities, its positive and active learning, teachers should according to the characteristics to Chinese martial arts teaching in the teaching, and emphasize on behavioral 
essentials, the purpose of the action, analyze the offensive and defensive means, make students deeply understand the connotation of the action, skill in mastering action and movements. Through the explanation and practice Chinese martial arts, causes the student to obtain the Chinese martial arts knowledge and technology, to establish a direct interest in learning Chinese martial arts [7]. There are so many ways to improve the learning interest of the Chinese martial arts, such as the teaching content of sectional renovation, long routine is brief, teachers should review more, and paragraph summary, praise more hard, progress faster students, lets the student have a feeling of success.

\section{Conclusion}

To grasp the generation of Chinese martial arts teaching in colleges and universities has important practical significance. At present, Chinese martial arts teaching is the teaching of public physical education in colleges and universities focus on routine of study, lack of Chinese martial arts education, Chinese martial arts public physical education teachers teaching quality improvement, Chinese martial arts teaching content is not suitable for public physical education, students' learning, and many other problems. In order to make the Chinese martial arts public physical education teaching is more able to adapt to social development and meet the demand of student's body and mind, Chinese martial arts colleges and universities should reform the teaching content, improve the students' learning interest, strengthens the Chinese martial arts teacher's teaching quality, training students' ability to use the learning motor skills, etc for the corresponding teaching reform.

\section{References}

[1] X. Y. Shi, Introduction to school of Chinese martial arts teaching how to out, Journal of Chinese martial arts science "fight" academic version, 2005, vol. 11, pp. 23-27.

[2] Q. H. Xu, Discuss about the present situation and the development of school Chinese martial arts, Journal of Jingmen technical college newspaper, 1999, vol. 3, pp. 34-37.

[3] Zh. L. Cai, Try to talk about school Chinese martial arts teaching research status and development trend, Guangzhou sports college journal, 2005, vol. 2, pp. 17-21.

[4] L. M. Liang, Try to talk about school of Chinese martial arts to carry out the road of innovation, Journal of Chinese martial arts, 2009, vol. 5, pp. 62-65.

[5] G. B. Zheng, School Chinese martial arts development difficult solutions, Journal of southwest institute for nationalities, 2002, vol. 8, pp. 43-46.

[6] G. L. Jiang, Curriculum under the background of the existing problems in the development of school Chinese martial arts and countermeasures, the road to success, 2009, vol. 6, pp. 54-57.

[7] T. H. Zhang, School of Chinese martial arts teaching reform and innovation, Journal of Huanggang normal university, 2008, vol. 11, pp. 42-45. 